\title{
DESCRIÇÃO DA COLONIZAÇÃO DE AEDES AEGYPTI NA REGIÃO DE SÃO JOSÉ DO RIO PRETO, SÃO PAULO
}

\author{
Francisco Chiaravalloti Neto
}

\begin{abstract}
O objetivo do trabalho é descrever a colonização da região pelo Aedes aegypti. Levantamento entomológico realizado em 1985 detectou a espécie em São José do Rio Preto. A dispersão do mosquito atingiu, até 1988, os 30 municípios da região. Nos distritos e aglomerados rurais, o primeiro foco do vetor foi encontrado em $1987 \mathrm{em} \mathrm{um}$ dos 29 existentes, dispersando-se para os demais até 1991. Os focos foram identificados, principalmente, através de pesquisas larvárias em locais com grande concentração de recipientes, e a maior freqüência de encontro de larvas de Ae. aegypti ocorreu em pneus, principais responsáveis por sua dispersão. Os focos foram identificados, basicamente, entre novembro e abril, períodos de maior incidência de chuvas. As delimitações dos focos mostraram que os principais recipientes infestados pelo mosquito nos domicílios foram os pneus e vasos de plantas. A conseqüência mais importante da presença do Ae. aegypti tem sido as ocorrências de epidemias de dengue.
\end{abstract}

Palavras-chaves: Aedes. Aedes aegypti. Colonização. Ecologia.

O Aedes aegypti é um mosquito originário da África. No Hemisfério Ocidental está estritamente associado com os seres humanos, sendo doméstico, antropofílico e procriandose em geral em recipientes artificiais 3 . É um grande problema para a população humana pois, juntamente com o Aedes albopictus, detém a capacidade de agir como vetor da febre amarela urbana, da dengue e da dengue hemorrágica, podendo causar sérias epidemias. As fêmeas adultas infectadas transmitem essas doenças ao se alimentarem de sangue humano, necessário para o desenvolvimento dos seus ovos 310.

Os mosquitos adultos podem ser reconhecidos por suas linhas prateadas no tórax em forma de lira e pelas listras brancas nos segmentos tarsais 16 . Na sua fase larvária os principais criadouros são os recipientes artificiais como pneus, caçambas, vasos, bebedouros de animais, latas, etc., isto é, objetos que retenham água3.

A erradicação do Ae. aegypti no Brasil, que já em 1934, extra-oficialmente, era uma das finalidades do Serviço de Combate à febre amarela, foi prevista no Decreto 8647 de 4 de fevereiro de 19426. Em 1947 as nações membros

Superintendência de Controle de Endemias (SUCEN) e Faculdades Integradas Riopretense (FIRP), São José do Rio Preto, SP.

Endereço para correpondência: Dr. Francisco Chiaravalloti Neto. R. Raul de Carvalho 2427, N.Sr ${ }^{\text {a }}$ Aparecida, 15025-300 São José do Rio Preto, SP, Brasil.

Recebido para publicação em 14/08/96. da Organização Pan-americana de Saúde resolveram erradicar o mosquito do Hemisfério Ocidental3. No Brasil, o último foco de $A e$. aegypti foi encontrado na zona rural do município de Santa Terezinha, BA, em 1955. O vetor é declarado erradicado do país em 19587. Outros dezoito países da América também realizaram a erradicação em épocas próximas19.

Em 1967, ocorre a primeira reintrodução do vetor no país, sendo em seguida eliminado7. Em 1976, a partir de um foco em Salvador, inicia-se a recolonização do Brasil pelo $A e$. aegypti12. Atualmente, ainda não foi detectada infestação domiciliar pelo Ae. aegypti somente nos Estados do Amazonas, Acre e Amapá10. Quase todos os países que também realizaram a erradicação do $A e$. aegypti foram reinfestados 20 .

No Estado de São Paulo, o Ae. aegypti foi reintroduzido em 1980 e 1981 , e os focos foram eliminados 17 . Com a finalidade de procurar pela presença do vetor nos municípios do estado, a Superintendência de Controle de Endemias, SUCEN, órgão vinculado à Secretaria de Estado da Saúde, realizou entre abril e maio de 1985 um levantamento da presença de criadouros de mosquitos em pontos estratégicos (PE), locais com grande concentração de recipientes como borracharias, postos de gasolina, depósitos de materiais de construção, ferros-velhos, etc15.

Nesse levantamento, pesquisaram-se e coletaram-se larvas de mosquitos nos recipientes encontrados nos PE, detectando-se focos de Ae. aegypti em alguns municípios do estado. 
Na região de São José do Rio Preto foram encontradas larvas do vetor apenas no município-sede, São José do Rio Preto16. A partir daí, outros municípios dessa região também se infestaram pelo Ae. aegypti.

O conhecimento do processo de ocupação da região pelo vetor é de fundamental importância para definir as medidas mais adequadas de vigilância e controle em momentos epidêmicos ou não, e mesmo para o equacionamento de medidas mais radicais, como a erradicação. Assim, o objetivo do trabalho foi o de descrever a colonização pelo Ae. aegypti dos 30 municípios da região de São José do Rio Preto, localizada na parte noroeste do Estado de São Paulo, ocorrida no período de 1985 a 1991.

\section{MATERIAL E MÉTODOS}

Com as informações coletadas no levantamento de criadouros do Ae aegypti realizado entre abril e maio de 1985 no município de São José do Rio Peto, foram obtidos o número e tipos de PE positivos (com encontro de larvas do vetor) e os tipos de recipientes positivos. Nos quarteirões onde a pesquisa inicial revelou a existência de PE positivos foram pesquisados os imóveis existentes, proporcionando o encontro de recipientes com larvas do Ae. aegypti em domicílios, detectando-se infestação domiciliar. Com as informações coletadas, obtiveram-se os tipos de imóveis e de recipientes positivos e calculou-se o Índice de Breteau (IB), índice de densidade larvária correspondente ao número de recipientes positivos para o vetor por 100 imóveis pesquisados 2 .

Após o primeiro levantamento e tendo como objetivo a detecção de focos e infestação domiciliar pelo vetor, foi montada uma rede de PE nos municípios não infestados da região de São José do Rio Preto para pesquisa sistemática de larvas de Ae. aegypti, como parte de estratégia estabelecida para todo o estado15. Infestação pelo vetor foi identificada, também, através de pesquisas larvárias decorrentes de notificação pela população da presença do mosquito ou de casos suspeitos de dengue ou febre amarela.

Nos municípios onde foi detectada a presença do mosquito, realizaram-se delimitações de focos (DF): pesquisas larvárias em raios de $200 \mathrm{~m}$ em torno dos focos iniciais. O encontro de larvas de $A e$. aegypti em domicílios nessas atividades caracterizou a existência de infestação domiciliar.
Em 29 distritos aglomerados rurais, localizados em 13 municípios da região, foram selecionados PE para a realização de pesquisas larvárias sistemáticas. Os encontros de PE positivos foram seguidos por DF para identificação de infestação domiciliar.

As informações apropriadas dos boletins de campo utilizados pela SUCEN para registro das várias atividades de pesquisas larvárias, no período de 1985 a 1991, foram utilizadas para descrição do histórico da colonização da região pelo vetor, e para obtenção dos tipos de PE e de recipientes positivos mais freqüentes, e os números médios de quarteirões, domićlíios e recipientes pesquisados e positivos e de larvas examinadas e positivas nas DF.

Calcularam-se as médias do IB para realização de comparações e também as médias dos IB parciais por recipientes para verificar o comportamento do mosquito com relação à ocupação de criadouros durante o processo de colonização da região pelo vetor.

As atividades de pesquisa larvária nos PE e nas DF foram realizadas em todos os imóveis que se enquadravam nas normas estabelecidas pela SUCEN, com exceção daqueles que se encontravam fechados no momento das pesquisas. Os dados obtidos, referentes à população de imóveis pesquisáveis, foram comparados entre si diretamente sem a utilização de testes estatísticos.

\section{RESULTADOS E DISCUSSÃO}

No levantamento realizado entre abril e maio de 1985, foram pesquisados $191 \mathrm{PE}$ em São José do Rio Preto. Das 455 coletas em recipientes, $25 \%$ foram positivas para o vetor, sendo a maior parte em pneus (72\%). As larvas positivas foram coletadas em $14 \mathrm{PE}$, principalmente nas borracharias, depósitos de pneus e recauchutadoras, e nas lojas e depósitos de materiais de construção (Tabela 1).

Os $14 \mathrm{PE}$ com larvas do vetor estavam distribuídos em 14 quarteirões do município. Para verificação de existência de infestação domiciliar, foram realizadas pesquisas larvárias em imóveis dessas quadras incluindo estabelecimentos comerciais ainda não pesquisados e domicílios. Coletaram-se larvas de Ae. aegypti em 23 recipientes, em sua maioria em borracharias, depósitos de pneus e recauchutadoras (65\%) e domicílios (27\%). O principal recipiente positivo encontrado foi $\mathrm{o}$ 
Chiaravalloti Neto F. Descrição da colonização de Aedes aegypti na região de São José do Rio Preto, São Paulo. Revista da Sociedade Brasileira de Medicina Tropical 30:279-285, jul-ago, 1997.

pneu (Tabela 2). Nos imóveis pesquisados encontrou-se IB igual a 23,0 recipientes positivos por 100 imóveis pesquisados.
A detecção do vetor apenas no municípiosede da região mostrou que a infestação era recente. A infestação domiciliar identificada no

Tabela 1 - Distribuição de recipientes positivos para larvas de Aedes aegypti, segundo tipo de pontos estratégicos (PE) pesquisados, São José do Rio Preto, SP, 1985.

\begin{tabular}{|c|c|c|c|c|c|c|c|}
\hline \multicolumn{3}{|c|}{ PE positivos } & \multicolumn{4}{|c|}{ № de Recipientes positivos por tipo } & \multirow{2}{*}{$\begin{array}{c}\text { Total } \\
\text { № }\end{array}$} \\
\hline Tipo* & $\mathrm{n}^{\mathrm{O}}$ & $\%$ & pneu & $\begin{array}{l}\text { lata e } \\
\text { garrafa }\end{array}$ & $\begin{array}{c}\text { tanque, tambor } \\
\text { e barril }\end{array}$ & outros & \\
\hline 1 & 6 & 43,0 & 11 & 0 & 1 & 0 & 12 \\
\hline 2 & 1 & 7,0 & 2 & 0 & 0 & 0 & 2 \\
\hline 3 & 4 & 29,0 & 0 & 0 & 1 & 4 & 5 \\
\hline 4 & 2 & 14,0 & 2 & 1 & 0 & 0 & 3 \\
\hline 5 & 1 & 7,0 & 3 & 0 & 0 & 0 & 3 \\
\hline Total & 14 & 100,0 & 18 & 1 & 2 & 4 & 25 \\
\hline$\%$ & - & - & 72,0 & 4,0 & 8,0 & 16,0 & 100,0 \\
\hline
\end{tabular}

4 = garagem de carros, ônibus e caminhões; 5 = ferro velho e desmanche.

Tabela 2 - Distribuição dos Recipientes positivos para larvas de Aedes aegypti , segundo tipo de imóveis comerciais e residenciais pesquisados, Sẫo José do Rio Preto, SP, 1985.

\begin{tabular}{|c|c|c|c|c|c|c|c|}
\hline \multicolumn{2}{|c|}{ Imóveis positivos } & \multicolumn{4}{|c|}{$\mathrm{N}^{\circ}$ de recipientes positivos por tipo } & \multicolumn{2}{|c|}{ Total } \\
\hline Tipo* & $\mathrm{n}^{\mathrm{o}}$ & pneus & lata e garrafa & vaso sanitário & bacia & $\mathrm{n}^{\mathrm{O}}$ & $\%$ \\
\hline 1 & 1 & 15 & 0 & 0 & 0 & 15 & 65,0 \\
\hline 2 & 1 & 1 & 0 & 0 & 0 & 1 & 4,0 \\
\hline 3 & 1 & 0 & 0 & 1 & 0 & 1 & 4,0 \\
\hline 4 & 2 & 4 & 1 & 0 & 1 & 6 & 27,0 \\
\hline Total & 5 & 20 & 1 & 1 & 1 & 23 & 100,0 \\
\hline$\%$ & - & 87,0 & 4,0 & 4,0 & 4,0 & 100,0 & - \\
\hline
\end{tabular}

4. Domicílios.

município de São José do Rio Preto, e também em mais 8 cidades do estado em 198515, colocou São Paulo ao lado de outras regiões brasileiras já reinfestadas pelo Ae. aegypti. A conseqüência mais importante foi a existência de risco de ocorrência de dengue e febre amarela em São Paulo. O levantamento realizado possibilitou uma visão geral do problema que serviu de base para a configuração de um programa de controle do vetor para o estado 15 .

Os encontros de focos e infestação domiciliar pelo vetor nos municípios da região por ano estão registrados na Tabela 3. Em dezembro de
1986 já existiam 9 (30,0\%) municípios com infestação domiciliar pelo Ae. aegypti, 22 $(73,3 \%)$ em dezembro de 1987 , e a totalidade em junho de 1988.

Em 73\% dos municípios o mosquito foi identificado apenas através de pesquisas larvárias positivas em PE, em 10\% através de pesquisas positivas realizadas em PE e simultaneamente devido às notificações da população, em 13\% devido apenas às notificações e $4 \%$ através de pesquisas positivas decorrentes de casos suspeitos de dengue ou febre amarela. A identificação de infestação domiciliar na grande

Tabela 3 - Municípios segundo ano de detecção de infestação domiciliar, região de São José do Rio Preto, SP, 1985 a 1988

\begin{tabular}{|c|c|c|c|c|}
\hline \multirow[t]{2}{*}{ Ano } & \multicolumn{3}{|c|}{ Municİpios } & \multirow{2}{*}{$\begin{array}{l}\text { № médio de habitantes* } \\
\text { (área urbana) }\end{array}$} \\
\hline & $\mathrm{n}^{\mathrm{O}}$ & nome & $\%$ acumulada & \\
\hline$\overline{1985}$ & 1 & São José do Rio Preto & 3,3 & 253.418 \\
\hline 1986 & 8 & $\begin{array}{l}\text { Bady Bassit, Cedral, Guapiaçu, Jaci, José Bonifácio, } \\
\text { Nova Aliança, Potirendaba, Tanabi }\end{array}$ & 30,0 & 7.441 \\
\hline 1987 & 13 & $\begin{array}{l}\text { Adolfo, Bálsamo, Icem, Mirassol, Mirassolândia, Monte Aprazível, } \\
\text { Orindiuva, Neves Paulista, Nova Granada, Palestina, } \\
\text { Planalto, Poloni, Zacarias }\end{array}$ & 73,3 & 7.236 \\
\hline 1988 & 8 & $\begin{array}{l}\text { Ibirá, Mendonça, Nipoã, Onda Verde, Paulo de Faria, Ubarana, } \\
\text { Uchoa, União Paulista }\end{array}$ & 100,0 & 3.491 \\
\hline
\end{tabular}

${ }^{*}$ Fonte:IBGE, 19948 
Chiaravalloti Neto F. Descrição da colonização de Aedes aegypti na região de São José do Rio Preto, São Paulo. Revista da Sociedade Brasileira de Medicina Tropical 30:279-285, jul-ago, 1997.

maioria dos municípios através de pesquisas em PE mostrou sua eficácia como mecanismo adequado de vigilância entomológica.

Nos distritos e aglomerados rurais, a primeira identificação de focos de Ae. aegypti com delimitação positiva ocorreu em agosto de 1987. Em dezembro de 1989, 86\% das localidades encontravam-se infestadas, e em 1991 todos os 29 distritos e aglomerados já estavam infestados domiciliarmente pelo mosquito.

A identificação de focos com infestação domiciliar pelo Ae. aegypti nas sedes municipais ocorreu em sua maioria entre os meses de novembro e abril $(76,6 \%)$. Nos distritos e aglomerados rurais, a identificação de focos e infestação domiciliar ocorreu em sua maioria entre os meses de novembro e fevereiro $(79,3 \%)$ (Tabela 4). Nesse processo notou-se uma relação importante com as precipitações pluviométricas. As detecções de focos com infestação domiciliar ocorreram em sua maioria entre novembro e abril, meses de maior incidência de chuvas na região14. Vários autores têm mostrado a existência de uma relação importante entre precipitação pluviométrica e presença e níveis de infestação de vetores, e mesmo casos de dengue1 4911.

Nos levantamentos de PE realizados nos municípios sem infestação domiciliar identificada, entre os anos de 1986 e 1988, os estabelecimentos positivos mais importantes foram as borracharias, depósitos de pneus e

Tabela 4 - Distribuição da identificação de focos e de infestação domiciliar por Aedes aegypti nas sedes municipais e nos distritos e aglomerados rurais, segundo meses do ano, região de São José do Rio Preto, SP, 1985 a 1991.

\begin{tabular}{|c|c|c|c|c|c|c|c|c|c|c|c|c|c|c|}
\hline \multirow{2}{*}{$\begin{array}{c}\text { № de localidades } \\
\text { e períodos }\end{array}$} & & \multicolumn{12}{|c|}{ Meses do ano } & \multirow[t]{2}{*}{ Tota } \\
\hline & & jan & fev. & mar & abr & mai & jun & jul & ago & set & out & nov & dez & \\
\hline Sedes & $\mathrm{n}^{\mathrm{O}}$ & 8 & 3 & 4 & 7 & 2 & 4 & 0 & 0 & 1 & 0 & 1 & 0 & 30 \\
\hline 1985 a 1988 & $\%$ & 26,7 & 10,0 & 13,3 & 23,4 & 6,7 & 13,3 & 0,0 & 0,0 & 3,3 & 0,0 & 3,3 & 0,0 & 100,0 \\
\hline Distr. e aglom. & $\mathrm{n}^{\mathrm{O}}$ & 7 & 9 & 0 & 0 & 1 & 2 & 0 & 1 & 2 & 0 & 5 & 2 & 29 \\
\hline 1987 a 1991 & $\%$ & 24,1 & 31,2 & 0,0 & 0,0 & 3,4 & 6,9 & 0,0 & 3,4 & 6,9 & 0,0 & 17,2 & 6,9 & 100,0 \\
\hline Total & $\mathrm{n}^{\mathrm{O}}$ & 15 & 12 & 4 & 7 & 3 & 6 & 0 & 1 & 3 & 0 & 6 & 2 & 59 \\
\hline 1985 a 1991 & $\%$ & 25,4 & 20,3 & 6,8 & 11,9 & 5,1 & 10,2 & 0,0 & 1,6 & 5,1 & 0,0 & 10,2 & 3,4 & 100,0 \\
\hline
\end{tabular}

recauchutadoras $(25,5 \%)$, seguidos por cemitérios (16,4\%), oficinas mecânicas $(14,5 \%)$ e lojas e depósitos de materiais de construção (12,7\%). Esses quatro tipos de estabelecimentos totalizaram 69,1\% dos locais positivos (Tabela 5). A positividade por tipo de criadouros nesta atividade é mostrada na Tabela 6 , sendo $57,4 \%$ deles pneus, seguidos por vasos $(16,0 \%)$. Os

Tabela 5 - Distribuição dos Pontos Estratégicos (PE) positivos para Aedes aegypti por tipos nos muncípios da região de São José do Rio Preto, SP, segundo ano, 1986 a 1988

\begin{tabular}{|c|c|c|c|c|c|c|}
\hline \multirow{2}{*}{ Ano } & \multirow{2}{*}{$\begin{array}{l}\text { Número de } \\
\text { PE positivos }\end{array}$} & \multicolumn{5}{|c|}{ Tipos de $\mathrm{PE}^{*}$} \\
\hline & & 1 & 2 & 3 & 4 & 5 \\
\hline 1986 & 12 & 3 & 4 & 1 & 1 & 3 \\
\hline 1987 & 33 & 9 & 4 & 5 & 6 & 9 \\
\hline 1988 & 10 & 2 & 0 & 1 & 2 & 5 \\
\hline Total & 55 & 14 & 8 & 7 & 9 & 17 \\
\hline$\%$ & 100,0 & 25,5 & 14,5 & 12,7 & 16,4 & 30,9 \\
\hline
\end{tabular}

Tabela 6 - Distribuição dos tipos de recipientes positivos para Aedes aegypti nos municípios da região de São José do Rio Preto, SP, segundo tipo de pontos estratégicos, 1986 a 1988.

\begin{tabular}{|c|c|c|c|c|c|}
\hline \multirow{2}{*}{$\begin{array}{c}\text { Tipo de } \\
\text { PE* }^{*}\end{array}$} & \multicolumn{3}{|c|}{ Tipos de recipientes } & \multicolumn{2}{|c|}{ Total } \\
\hline & pneu & vaso & outros & $\mathrm{n}^{\mathrm{O}}$ & $\%$ \\
\hline 1 & 29 & 0 & 1 & 30 & 31,9 \\
\hline 2 & 5 & 3 & 3 & 11 & 11,7 \\
\hline 3 & 3 & 0 & 8 & 11 & 11,7 \\
\hline 4 & 0 & 12 & 1 & 13 & 13,8 \\
\hline 5 & 17 & 0 & 12 & 29 & 30,9 \\
\hline Total & 54 & 15 & 25 & 94 & 100,0 \\
\hline$\%$ & 57,4 & 16,0 & 26,6 & 100,0 & - \\
\hline
\end{tabular}


pneus foram os principais responsáveis pela dispersão passiva do mosquito através de intenso comércio realizado com este tipo de material, entre os municípios da região, entre a região e os demais municípios do Estado de São Paulo e mesmo com outras localidades brasileiras.
Na Tabela 7 são apresentadas informações relativas às DF com encontro de infestação domiciliar entre 1986 e 1988. Verifica-se que o número médio de quarteirões, domicílios, recipientes e larvas pesquisados e positivos são crescentes entre os anos de 1986 e 1988. Esses

Tabela 7 - Números médios de quarteirões, imóveis, recipientes e larvas pesquisados e positivos para Aedes aegypti em delimitações de focos (DF), segundo ano, região de São José do Rio Preto, SP, 1986 a 1988.

\begin{tabular}{|c|c|c|c|c|c|c|c|c|c|}
\hline \multirow[t]{2}{*}{ Ano } & \multirow{2}{*}{$\begin{array}{l}\text { № } \\
\text { de } \\
\text { DF }\end{array}$} & \multicolumn{2}{|c|}{$\begin{array}{c}\text { № médio de } \\
\text { quarteirões por DF }\end{array}$} & \multicolumn{2}{|c|}{$\begin{array}{c}\text { № médio de } \\
\text { domicílios por DF }\end{array}$} & \multicolumn{2}{|c|}{$\begin{array}{l}\text { № médio de } \\
\text { repientes por DF }\end{array}$} & \multicolumn{2}{|c|}{$\begin{array}{l}\text { № médio de } \\
\text { larvas por DF }\end{array}$} \\
\hline & & pesquis. & positivos & pesquis. & positivos & pesquis. & positivos & pesquis. & positivos \\
\hline 1986 & 8 & 15 & 5 & 211 & 8 & 644 & 11 & 152 & 43 \\
\hline 1987 & 13 & 37 & 8 & 462 & 21 & 1445 & 23 & 296 & 101 \\
\hline 1988 & 8 & 74 & 32 & 832 & 65 & 2831 & 72 & 1404 & 935 \\
\hline
\end{tabular}

dados indicam uma colonização cada vez maior do mosquito nos municípios da região.

Na Tabela 8, são apresentadas as médias dos IB, total e por recipientes calculadas a partir das DF realizadas para o período de 1986 a 1988. O IB aumentou de 4,6 em 1986 para 10,0 em 1988. Avaliando-se os IB parciais por recipientes, em 1986 apenas dois tipos de recipientes tiveram participação maior que $10 \%$ do valor do IB total, pneus e vasos de plantas; em 1987 três tipos de recipientes apresentaram valores maiores que $10 \%$ do IB total, pneus, vasos de plantas e latas e garrafas; e em 1988, outros tipos de recipientes que não os citados passam também a ter maior importância. Com o passar do tempo, verificou-se que a ocupação inicial, nos domicílios, mais restrita a pneus e vasos de plantas, foi ampliada para latas e garrafas e outros tipos de recipientes, mostrando também a evolução da colonização do mosquito na região. Nessas DF, nota-se o papel ocupado pelos pneus e vasos de plantas na infestação domiciliar pelo $A e$ a aegypti, que foi responsável por $86,3 \%$ do valor do IB em 1986, 63,6\% em 1987 e 49,2\% em 1988.

Num prazo pouco superior a 3 anos, de 1985 a 1988, o mosquito, que foi identificado no município-sede da região, propagou-se

Tabela 8- Indices médios de Breteau (IB) calculados a partir de delimitações de focos (DF), segundo ano, região de São José do Rio Preto, SP, 1986 a 1988

\begin{tabular}{|c|c|c|c|c|c|c|c|c|c|c|c|c|c|}
\hline \multirow{3}{*}{ Ano } & \multirow{3}{*}{$\begin{array}{l}\mathrm{N}^{\circ} \\
\text { de } \\
\text { DF }\end{array}$} & \multirow{2}{*}{\multicolumn{2}{|c|}{$\begin{array}{c}\text { IB } \\
\text { Total }\end{array}$}} & \multicolumn{10}{|c|}{ IB por tipo de recipientes } \\
\hline & & & & \multicolumn{2}{|c|}{ pneu } & \multicolumn{2}{|c|}{ Vaso } & \multicolumn{2}{|c|}{ lata-garrafa } & \multicolumn{2}{|c|}{ tambor-tanque } & \multicolumn{2}{|c|}{ outros tipos } \\
\hline & & valor & $\%$ & valor & $\%$ & valor & $\%$ & valor & $\%$ & valor & $\%$ & valor & $\%$ \\
\hline 1986 & 8 & 4,6 & 100,0 & 1,2 & 25,3 & 2,8 & 61,0 & 0,4 & 9,2 & 0,1 & 2,2 & 0,1 & 2,3 \\
\hline 1987 & 13 & 5,0 & 100,0 & 2,2 & 43,1 & 1,0 & 20,5 & 1,0 & 20,2 & 0,4 & 8,1 & 0,4 & 8,1 \\
\hline 1988 & 8 & 10,0 & 100,0 & 3,0 & 29,9 & 2,9 & 29,3 & 2,0 & 19,9 & 0,3 & 2,9 & 1,8 & 18,0 \\
\hline
\end{tabular}

para todos os outros municípios. Em um período de 4 anos, de 1987 a 1991, processo semelhante ocorreu com os distritos e aglomerados rurais. As medidas de controle adotadas não foram suficientes para interromper a sua evolução, sendo que o mosquito encontrou ambiente bastante favorável tanto na oferta de criadouros como nas condições climáticas adequadas: altas temperaturas e principalmente grande incidência de chuva entre os meses de novembro e abril14.

A colonização da região pelo Ae. aegypti se deu no sentido da maior cidade (São José do Rio Preto) para os municípios menores, e destes para os distritos e aglomerados rurais. As evidências deste processo estão nos números médios de habitantes decrescentes das áreas urbanas dos municípios infestados entre os anos de 1985 e 1988 (Tabela 3), e no fato de que a primeira identificação de infestação domiciliar em um aglomerado rural em agosto de 1987 ter ocorrido quando 70\% das sedes municipais já se encontravam infestadas. Essa dispersão do mosquito se deu, além das condições climáticas favoráveis, em função de intercâmbio econômico entre São José do Rio Preto e os demais municípios e entre estes e os distritos e aglomerados rurais, tendo os pneus 
desempenhado papel importante neste processo.

Após a ampla colonização da região pelo Ae. aegypti, ocorrida entre 1985 e 1991, seus municípios também passaram a ser colonizados por outra espécie vetora da dengue e da febre amarela, o Ae. albopictus. Esse vetor foi detectado pela primeira vez na região em 1993 em São José do Rio Preto, e até dezembro de 1994 já estava presente em pelo menos mais 18 municípios5. A presença simultânea das duas espécies na região deverá ser motivo de estudos posteriores para avaliação da interação entre elas.

Com a presença de mosquitos vetores da dengue e da febre amarela, a região passou a sofrer risco de ocorrência de epidemias. Em 1986, a circulação de um caso importado de febre amarela em São José do Rio Preto desencadeou um processo de vacinação de emergência da população. A partir de 1991, passaram a ocorrer epidemias cada vez mais importantes de dengue. Neste ano foram confirmados laboratorialmente 157 casos autóctones de dengue em 7 municípios da região e, até junho de 1996, foram confirmados 1023 casos em 17 municípios, notando-se o aumento progressivo do número de casos e o do número de municípios envolvidos, colocando a região sob o risco de ocorrência de dengue hemorrágico13 18 .

\section{SUMMARY}

The aim of this study is to describe the colonization by the Aedes aegypti in the region. $A$ survey carried out in 1985 detected the species in São José do Rio Preto. The mosquito has spread and reached the 30 counties of the region till 1988. In the district and rural areas, the first vector focus was found out in 1987 in one of the 29 districts and rural areas, having spread to the others till 1991. The foci have been mainly identified through larval researches in locations with a great concentration of containers, and the greatest occurrence of larvae of Aedes aegypti has been in tires, the most frequent means of spread. The foci have been mainly identified between November and April, periods of greater incidence of rains. The delimits of foci showed that the containers which were mostly infested by the mosquito in homes have been tires and vases of plants. The most important consequence of the presence of Aedes aegypti has been the occurrence of dengue epidemics.

Key-words: Aedes., Aedes aegypti. Colonization. Ecology.

\section{REFERÊNCIAS BIBLIOGRÁFICAS}

1. Bellorin EG. La participacion comunitária en el control del Aedes aegypti en Honduras durante 1989 a 1990. Honduras [mimeografado], 1991.

2. Breteau H. La fièvre jaune en Afrique- Ocidentale Française. World Health Organization Bulletin, 11:453-481,1954.

3. Center for Disease Control. Biology and Control of Aedes aegypti. Center for Disease Control, Atlanta, 1979.

4. Chiaravalloti Neto F. Aedes aegypti na Região de São José do Rio Preto, Estado de São Paulo. Tese de mestrado, Faculdade de Saúde Pública da Universidade de São Paulo, São Paulo, SP, 1993.

5. Chiaravalloti Neto F, Costa AIP, Cardoso Jr RP, Scandar SAS, Soares MRD. Descrição da colonização de Aedes albopictus (Diptera: Culicidae) na Região de São José do Rio Preto, SP, no período de 1991 a 1994. In: Resumos do XXXI Congresso da Sociedade Brasileira de Medicina Tropical, São Paulo p. 208, 1995.
6. Chieffi PP. Algumas questões decorrentes da reintrodução do Aedes aegypti no Brasil no Brasil. Cadernos de Saúde Pública, Rio de Janeiro 1:193199, 1985.

7. Franco O. História da febre amarela no Brasil. Ministério da Saúde, Rio de Janeiro, 1976.

8. Fundação Instituto Brasileiro de Geografia e Estatística. IBGE. Censo demográfico 1991. Resultados do universo relativo às características da população e dos domicílios (vol 21). Fundação Instituto Brasileiro de Geografia e Estatística, Rio de Janeiro, 1994.

9. Koopmann JS, Prevots DR, Marin MAV, Dantes HG, Aquino MLZ, Longini Jr IM, Amor, JS. Determinants and predictors of dengue infeccion in Mexico. American Journal of Epidemiology 133:1168-1178, 1991.

10. Ministério da Saúde. Fundação Nacional de Saúde. Manual de Dengue - Vigilância Epidemiológica e Atenção ao Doente. Ministério da Saúde, Brasília, 1996. 
11. Moore GG, Cline BL, Ruiz-Tibén E, Lee D, RomneyJoseph H, Rivera-Correa E. Aedes aegypti in Puerto Rico:environmental determinants of larval abundance and relation to dengue virus transmission. American Journal of Tropical Medicine and Hygiene 27:1225-1231, 1978.

12. Nobre A, Antezana D, Tauil PL. Febre amarela e dengue no Brasil:epidemiologia e controle. Revista da Sociedade Brasileira de Medicina Tropical 27:59-65, 1994 .

13. Organização Pan-americana da Saúde. Diretrizes relativas à prevenção e ao controle da dengue e da dengue hemorrágica nas Américas. Relatório da Reunião sobre diretrizes para a dengue. Organização Pan-americana da Saúde, Washington [mimeografado], 1991.

14. Secretaria de Estado daAgricultura eAbastecimento. Divisão Regional Agrícola de São José do Rio Preto. Boletins de precipitação pluviométrica e temperatura. Secretaria de Estado da Agricultura e Abastecimento, São José do Rio Preto, 1985 a 1991.

15. Secretaria de Estado da Saúde. Superintendência de Controle de Endemias. Programa de Controle do Aedes aegypti no Estado de São Paulo. Secretaria de Estado da Saúde, São Paulo [mimeografado], 1985.

16. Secretaria de Estado da Saúde. Centro de Vigilância Epidemiológica. Manual de Vigilância Epidemiológica da dengue e da febre amarela. Secretaria de Estado da Saúde, São Paulo, 1987.

17. Secretaria de Estado da Saúde. Vigilância epidemiológica do dengue e da febre amarela no Estado de São Paulo. Secretaria de Estado da Saúde, São Paulo [mimeografado], 1991a.

18. Secretaria de Estado da Saúde. Superintendência de Controle de Endemias. Plano de emergência para o controle dos vetores da dengue e da febre amarela no verão de 1991/1992. Secretaria de Estado da Saúde, São Paulo [mimeografado], $1991 \mathrm{~b}$.

19. Tauil PL. O problema do Aedes no Brasil. Revista da Sociedade Brasileira de Medicina Tropical 19:13, 1986.

20. Tauil PL. Aedes aegypti e Aedes albopictus no Brasil. Revista da Sociedade Brasileira de Medicina Tropical 22:55, 1989. 